\title{
A Rough-Fuzzy HSV Color Histogram for Image Segmentation
}

\author{
Alessio Ferone ${ }^{1}$, Sankar Kumar $\mathrm{Pal}^{2}$, and Alfredo Petrosino ${ }^{1}$ \\ 1 DSA - Universitá di Napoli Parthenope, 80143 Napoli, Italy \\ 2 Machine Intelligence Unit, Indian Statistical Institute, Kolkata 700 108, India
}

\begin{abstract}
A color image segmentation technique which exploits a novel definition of rough fuzzy sets and the rough-fuzzy product operation is presented. The segmentation is performed by partitioning each block in multiple rough fuzzy sets that are used to build a lower and a upper histogram in the HSV color space. For each bin of the lower and upper histograms a measure, called $\tau$ index, is computed to find the best segmentation of the image. Experimental results show that the proposed method retains the structure of the color images leading to an effective segmentation.
\end{abstract}

Keywords: Image segmentation, Color Image Histogram, Rough Sets, Fuzzy Sets.

\section{Introduction}

Color image segmentation is one of the most challenging tasks in image processing, being the basic pre-processing step of many computer vision and pattern recognition problems.

Among the others, the most used approaches are represented by histogram based techniques due to the fact they need no a-priori information about the image. The task consists in finding clusters corresponding to regions of uniform colors, identified by peaks in the histogram. The task is complicated in color images, being characterized by three dimensional scatterograms, that make more difficult the search for peaks, either in the whole histogram or in each color channel independently. Also, typically they do not take into account the spatial correlation between adjacent pixels, while images usually show this property.

The approach reported here bases its rationale on Granular Computing, based on the concept of information granule, that is a set of similar objects that can be considered as indistinguishable. Partition of an universe in granules gives a coarse view of the universe where concepts, represented as subsets, can be approximated by means of granules. In this framework, rough set theory can be regarded to as a family of methodologies and techniques that make use of granules 89. The focus of rough set theory is on the ambiguity caused by limited discernibility of 
objects in the domain of discourse. Granules are formed as objects and are drawn together by the limited discernibility among them. Granulation is of particular interest when a problem involves incomplete, uncertain or vague information. In such cases, precise solutions can be difficult to obtain and hence the use of techniques based on granules can lead to a simplification of the problem at hand.

At the same time, multivalued logic can be applied to handle uncertainty and vagueness in information system, the most famous of which is fuzzy sets theory [16. In this framework, uncertainty is modelled by means of functions that define the degree of belonginess of an object to a given concept. Hence membership functions of fuzzy sets enable efficient handling of overlapping classes.

Some researches already follow this approach. Cheng et al. 2] employed a fuzzy homogeneity approach to extract homogeneous regions in a color image. The proposed method introduces the concept of homogram built considering intensity variation in pixel neighborhood. In [5] the concept of encrustation of the histogram (histon), which is a contour plotted on the top of each primary color histogram, is presented. In a rough-set theoretic sense, the histon represents the upper approximation of the color regions, that is a collection of pixels possibly belonging to the same region, while the histogram represents the lower approximation. An histogram-based technique is employed on the histon to obtain the final segmentation. Mushrif and Ray [6] presented a segmentation scheme, based on the concept of histon [5], which employs the roughness index. Roughness is large when the boundary contains a large number of elements, hence it will be smaller in the boundary between two objects and larger in region with uniform color.

The novelty of our approach resides on the hybrid notion of rough fuzzy sets that comes from the combination of these two models of uncertainty (fuzzy and rough) to exploit, at the same time, properties like coarseness, by handling rough sets [8], and vagueness, by handling fuzzy sets [16]. In this framework, rough sets embody the idea of indiscernibility between objects in a set, while fuzzy sets model the ill-definition of the boundary of a sub class of this set. Marrying both notions leads to consider, as instance, approximation of sets by means of similarity relations or fuzzy partitions. The rough fuzzy synergy is hence adopted to better represent the uncertainty in granular computation. Specifically, we present a histogram based technique tat exploits a generalized definition of rough-fuzzy sets, i.e. an hybridization of rough sets and fuzzy sets, and a particular operation called rough-fuzzy product in the HSV color space.

\section{Rough Fuzzy Color Histogram}

Let us consider an image $I$ defined over a set $U=[0, \ldots, H-1] \times[0, \ldots W-1]$ of picture elements, i.e. $I: u=\left(u_{x}, u_{y}\right) \in U \rightarrow[h(u), s(u), v(u)]$. We shall introduce the Image Partition as 
Definition 1. Let us consider a grid, superimposed on the image, whose cells $Y_{i}$ are of dimension $w \times w$. Given a pixel $u$, whose coordinates are $u_{x}$ and $u_{y}$, and a cell $Y_{i}$ of the grid, whose coordinates of its upper left point are $x\left(Y_{i}\right)$ and $y\left(Y_{i}\right)$, $u$ belongs to $Y_{i}$ if $x\left(Y_{i}\right) \leq u_{x} \leq x\left(Y_{i}\right)+w-1$ and $y\left(Y_{i}\right) \leq u_{y} \leq y\left(Y_{i}\right)+w-1$.

The set of all $Y_{i}$ constitutes an Image Partition, $\mathcal{Y}$, over $I$.

Different values of $w$ yield different partitions $\mathcal{Y}$ of the same image. For instance, given a partition $\mathcal{Y}^{i}$, other partitions can be obtained by a rigid translation in the directions of $0^{\circ}, 45^{\circ}$ and $90^{\circ}$ degrees of $w-1$ pixels, so that for each partition a pixel belongs to a shifted version of the same cell $Y_{j}^{i}$.

If we consider four cells, $Y_{j}^{1}, Y_{j}^{2}, Y_{j}^{3}$ and $Y_{j}^{4}$ belonging to four partitions $\mathcal{Y}^{1}$ $\mathcal{Y}^{2} \mathcal{Y}^{3} \mathcal{Y}^{4}$, then there exists a pixel $u$ with coordinates $\left(u_{x}, u_{y}\right)$ such that $u$ belongs to the intersection of $Y_{j}^{1}, Y_{j}^{2}, Y_{j}^{3}$ and $Y_{j}^{4}$ [11.

$$
Y_{j}^{1,2,3,4}=Y_{j}^{1} \cap Y_{j}^{2} \cap Y_{j}^{3} \cap Y_{j}^{4}
$$

The image is firstly partitioned in non-overlapping $k$ blocks $X_{h}$ of dimension $m \times m$, such that $m \geq w$, that is $X=\left\{X_{1}, \ldots, X_{k}\right\}$ and $k=H / m+K / m$. Considering each image block $X_{h}$, a pixel in the block can be characterized by two values $h_{\text {inf }}(u)$ and $h_{\text {sup }}(u)$ computed, for each pixel $u$ belonging to a block $X_{h}$, as

$$
\begin{aligned}
h_{\mathrm{sup}}(u) & =\sup \left\{h_{m}^{1}(u), h_{m}^{2}(u), h_{m}^{3}(u), h_{m}^{4}(u)\right\} \\
h_{\mathrm{inf}}(u) & =\inf \left\{h_{M}^{1}(u), h_{M}^{2}(u), h_{M}^{3}(u), h_{M}^{4}(u)\right\}
\end{aligned}
$$

where $h_{m}^{i}(u) i=2,3,4$ are obtained by translating $h_{m}^{1}(u)$ in the direction of 0 , 45 and 90 degrees. For instance, for $w=2$ and a generic $j-t h$ cell of the $i-t h$ partition, we have:

$$
\begin{aligned}
& h_{m}^{i}(u)=\inf \left\{\left(u_{x}+a, u_{y}+b\right) \mid a, b=0,1\right\} \\
& h_{M}^{i}(u)=\sup \left\{\left(u_{x}+a, u_{y}+b\right) \mid a, b=0,1\right\}
\end{aligned}
$$

Let us now consider the HSV color space represented by a cone and a segment $[\theta, \theta+\Delta \theta-1]$ on the maximum circumference, where $0 \leq \theta \leq 359$ and $\left[\Delta \theta_{\min } \leq\right.$ $\left.\Delta \theta \leq \Delta \theta_{\max }\right]$ is the segment dimension. This interval contains a certain amount of colors. In particular, if we imagine to cut the HSV cone in wedges, each one contains all the possible combination of saturation and value given a portion of hue. Our goal is to describe each wedge using the blocks of the image, under the assumption that blocks with similar colors will fall in the same wedge.

Definition 2. Each block $X_{h}$, of dimension $m \times m$, is characterized by a minimum and a maximum hue value 


$$
\begin{aligned}
h_{m} & =\min \left\{h_{\mathrm{sup}}(u) \mid u \in X_{h}\right\} \\
h_{M} & =\max \left\{h_{\mathrm{inf}}(u) \mid u \in X_{h}\right\}
\end{aligned}
$$

defining a hue Interval $\left[h_{m}, h_{M}\right]$ that can be:

1. totally contained into a wedge of dimension $\Delta \theta$ (i.e. $\theta \leq h_{m} \leq h_{M}<\Delta \theta+\theta$ ),

2. partially contained into a wedge (i.e. $\theta \leq h_{m}$ or $h_{M}<\Delta \theta+\theta$ ),

3. not contained at all.

Hence, we can describe the wedge by means of two sets of blocks.

Definition 3. The $\mathbf{L}$-set is the set of blocks whose interval $\left[h_{m}, h_{M}\right]$ are totally contained into the wedge. The $\mathbf{U}$-set is the set of blocks whose $\left[h_{m}, h_{M}\right]$ are partially contained into the wedge.

Now consider a wedge of dimension $\left[\theta_{i}, \theta_{i}+\Delta \theta-1\right], i=0, \ldots, 359$ moving on the hue circle towards increasing hue values, starting from $\theta_{1}=0$. At each step the wedge is shifted by an offset x, i.e. $\theta_{i+1}=\theta_{i}+\mathrm{x}$, and the $L$-set and $L$-set of the wedge are computed. This procedure, shown in Algorithm 1, yields two histograms, the L-Histogram and the U-Histogram of the image.

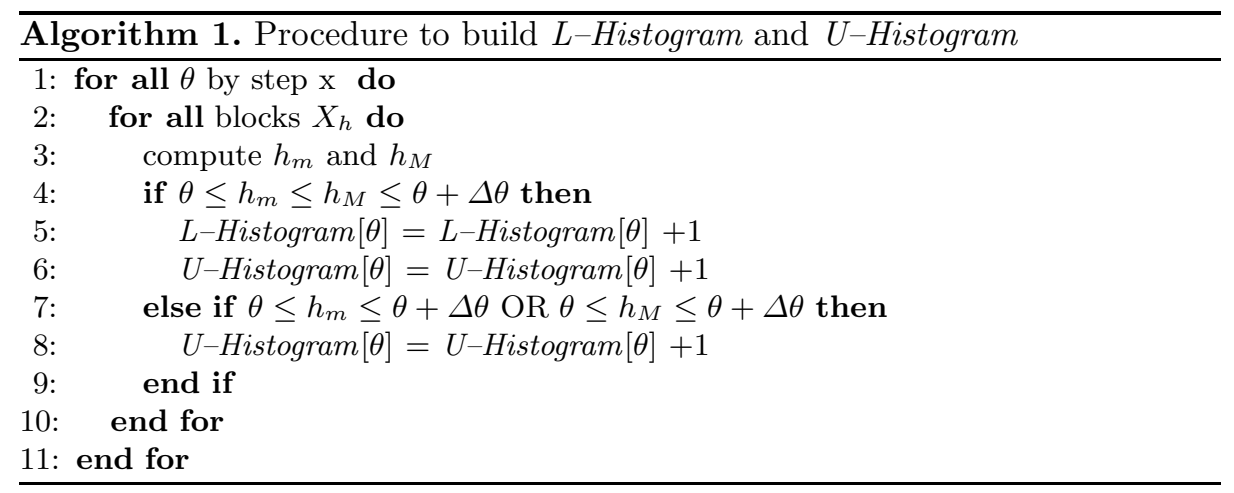

Repeating the same procedure for each wedge dimension $\theta_{\min } \leq \theta \leq \theta_{\max }$, many histograms are produced according to the possible values of $\theta$. Figure 1 and 2 depict respectively the $L$-Histogram and the $U$-Histogram of Figure 1.

It should be reminded that, if for a given pixel the saturation equals 0 , the hue component is undefined and the pixel is characterized only by the value component, i.e. only by its gray level intensity. To overcome this problem, it is possible to exclude all the pixels with a saturation value lower than a given threshold $\epsilon$ and segment them separately (for instance employing a segmentation algorithm for gray scale images). 


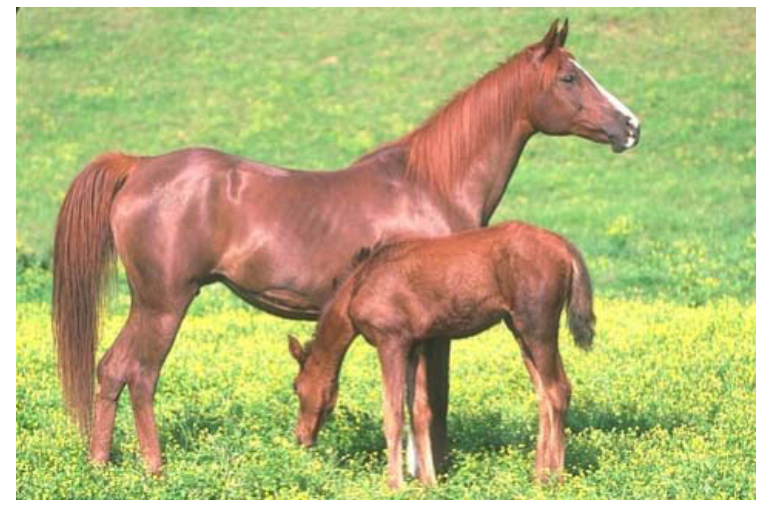

Fig. 1. Example image

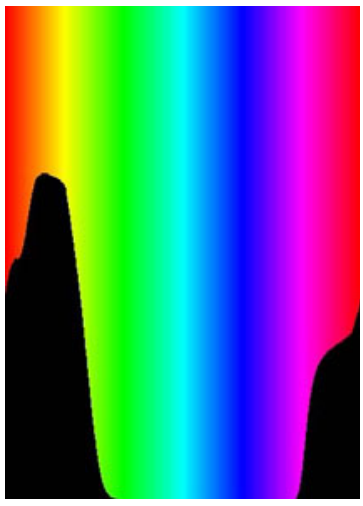

(a)

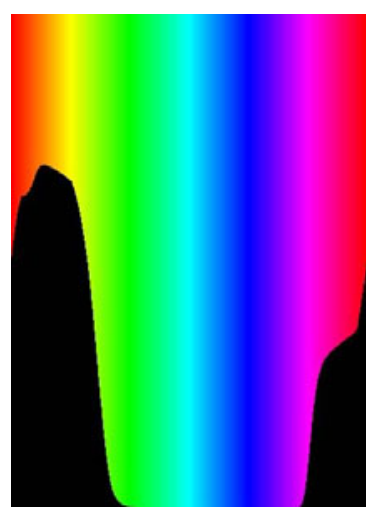

(b)

Fig. 2. a) L-Histogram and b) U-Histogram

\section{Image Segmentation by Rough Fuzzy Color Histogram}

The segmentation of a color image is performed in the HSV color space by choosing the wedges that are better represented employing the blocks of the image. The choice is guided by the accuracy of the wedge, i.e. the $i$-th wedge gets an accuracy computed by means of the corresponding bin in the L-Histogram and U-Histogram

$$
\alpha_{i}=\frac{L-\operatorname{Histogram}(i)}{U-\operatorname{Histogram}(i)}
$$

Clearly, this can not be the only discriminant index to obtain a good segmentation. First of all due to the accuracy, as computed in eq. 2, that does not take into account the number of blocks, and hence the number of pixels contained into the wedge, but only their ratio. Moreover, using only the accuracy does not 
take into account saturation and value of each pixel. The first problem is tackled by weighting the accuracy of each wedge by the fraction of pixels whose hue value belongs to the wedge, i.e.

$$
\gamma_{i}=1-\frac{N_{w e d g e}(i)}{N_{\text {tot }}(I)}
$$

where $N_{\text {wedge }}(i)$ represents the number of pixels whose hue value belongs to the wedge and $N_{\text {tot }}(I)$ represents the number of pixels of the image $I$.

Provided that regions of uniform colors are searched into the image, we need an index to measure the color uniformity of the pixels belonging to the the wedge and then use this index to weight the accuracy. To this aim we propose to employ a measure of the dispersion of the pixels falling into a wedge with respect to saturation and value. A region characterized by uniform color will present a narrow scatter, while a region characterized by non uniform colors will have a sparse scatter. To compute the compactness of saturation and value into the $i$-th wedge, we propose the following index

$$
\delta_{i}=\frac{1}{N_{\text {wedge }}(i)} \times \sqrt{\sum_{x \in i-\text { thwedge }}\left(x-\mu_{i}\right)^{T}\left(x-\mu_{i}\right)}
$$

where $x=\left[x_{\text {saturation }}, x_{\text {value }}\right]$. This index can be considered as the weighted squared root of the track of the covariance matrix. The final index, $\tau_{i}$, is computed by composing $\alpha_{i}, \gamma_{i}$ and $\delta_{I}$ indices (eqs. 2, 3] and 4)

$$
\tau_{i}=\alpha_{i} \times\left(w_{1} \times \gamma_{i}+w_{2} \times \delta_{i}\right)
$$

where $w_{1}$ and $w_{2}$, with $w_{1}+w_{2}=1$, are parameters used to weight the fraction of pixels falling into a wedge and the saturation-value dispersion, respectively. A higher value for $w_{1}$ will lead to wedges comprising few pixels characterized by a low saturation-value dispersion, whilst a higher value for $w_{2}$ will produce wider wedges, with a larger number of pixels presenting a lower saturationvalue dispersion. The index $\tau$, computed for all the wedges, is used to segment the image. Firstly, the wedge with the highest $\tau$ value is selected as the region better represented into the image. Next, all the wedges that intersect the first one are removed to avoid overlapping regions. For instance, consider $s_{i}$ the wedge with the highest $\tau$ value corresponding to the hue segment $q_{s_{i}}, q_{s_{i}}+q t-1$, then all the wedges $s_{j}$ such that $q_{s_{i}} \leq q_{s_{j}}+\tilde{q} t-1<q_{s_{i}}+q t-1$, with $\tilde{q} t$ varying in $\left[q t_{\min }, q t_{\max }\right]$, are removed. Next, the wedge with the highest $\tau$ value, among those not removed in the previous step, is selected, and so on until no more wedges are left.

\section{Experimental Results}

To assess the performance of the proposed method, we employed the Probabilistic Rand Index (PRI) 15] that counts the fraction of pairs of pixels whose 
labellings are consistent between the computed segmentation and the ground truth, averaging across multiple ground truth segmentations to account for scale variation in human perception. For each image, the quality of the segmentation is evaluated by comparing it with all the available segmentations of the same image.

The performance of the proposed algorithm were tested on the 100 color test images of "The Berkeley Segmentation Dataset" 4. Threshold has been fixed to $\epsilon=0.2$; all the pixels presenting a saturation value lower than $\epsilon$ have been segmented by employing another threshold $\eta=0.5$, i.e., pixels are labelled as "white" if their value component is greater than $\eta$, as "black" otherwise. A larger granule dimension allows to produce wedges able to enclose more similar hues so to suppress small hue variations, while smaller granule dimension tends to better differentiate between similar hues. A larger granule size can be useful to segment images that show larger hue variance and hence obtain better PRI. Parameters $w_{1}$ and $w_{2}$ can be used to obtain distinct segmentations by weighting the importance of the number of pixels into the wedge with respect to the saturation-value dispersion. Higher values of $w_{1}$ mean that wedges enclosing few pixels are privileged, while higher values of $w_{2}$ privilege wedges characterized by higher saturation-value dispersion.

Figure 3 shows an example of segmentation of two test images of the BSD. Segmentation in Figure 3(a) obtained with parameters $w_{1}=0.6 w_{2}=0.4$
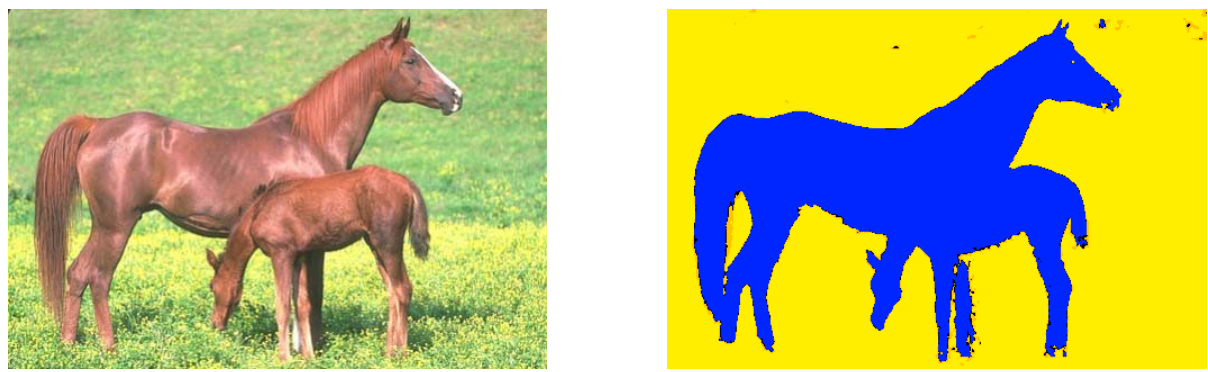

(a)
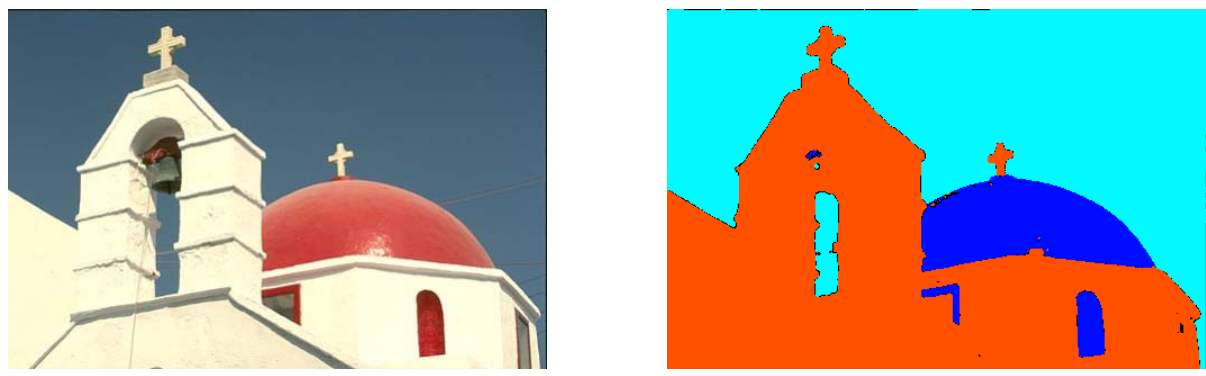

(b)

Fig. 3. Segmentation for images 113044 (a) and 118035 (b) 
and granule dimension $w=2$, produces $P R I=0.774117$. Segmentation in Figure $3(\mathrm{~b})$ obtained with parameters $w_{1}=0.7 w_{2}=0.3$ and granule dimension $w=2$, produces $P R I=0.870635$.

Table 1 summarizes results obtained with different parameter configurations in terms of mean PRI computed over the 100 color images adopted for testing the algorithm. It turns out that best results are obtained using small granule dimensions and giving importance to the number of pixels over the saturationvalue dispersion. Here we want to point out that, although this configuration gives the best results on average, this does not imply that good results could not be obtained for single images employing different values.

Table 1. Mean PRI values for the 100 test images of the BSD

\begin{tabular}{|c|c|c|c|}
\hline Granule dimension $w$ & $w_{1}=0.8, w_{2}=0.2$ & $w_{1}=0.6, w_{2}=0.4$ & $w_{1}=0.5, w_{2}=0.5$ \\
\hline 2 & 0.678028 & 0.663410 & 0.654179 \\
\hline 4 & 0.661959 & 0.636016 & 0.624948 \\
\hline 8 & 0.640885 & 0.621997 & 0.619233 \\
\hline 16 & 0.623986 & 0.613345 & 0.609314 \\
\hline 32 & 0.618413 & 0.601521 & 0.590546 \\
\hline
\end{tabular}

\section{Conclusions}

Color image segmentation is of particular interest because the huge amount of information held by colors can make the task very difficult to perform, although it can give fundamental information about the image to be analyzed. In this paper we have presented a segmentation technique, performed in the HSV color space, that exploits peculiarities of rough-fuzzy sets and, in particular, a feature extraction operation called rough-fuzzy product. The proposed method, tested against a typical human hand made segmentation dataset, have shown good segmentation capabilities although more research is needed to obtain good average performance. Ongoing work is devoted to consider spatial relationship between blocks to increase the performance of the algorithm.

\section{References}

1. Chapron, M.: A new chromatic edge detector used for color image segmentation. In: Proc. 11th Int. Conf. on Pattern Recognition, vol. 3, pp. 311-314 (1992)

2. Cheng, H.D., Jiang, X.H., Wang, J.: Color image segmentation based on homogram thresholding and region merging. Pattern Recognition 35, 373-393 (2002)

3. Li, S.Z.: Markov Random Field Modeling in Computer Vision, Kunii, T.L. (ed.). Springer, Berlin (1995)

4. Martin, D., Fowlkes, C., Tal, D., Malik, J.: A Database of Human Segmented Natural Images and its Application to Evaluating Segmentation Algorithms and Measuring Ecological Statistics. In: Proc. 8th Int Conf. Computer Vision, vol. 2, pp. 416-423 (2001) 
5. Mohabey, A., Ray, A.K.: Rough set theory based segmentation of color images. In: Proc. 19th Internat. Conf. NAFIPS, pp. 338-342 (2000)

6. Mushrif, M.M., Ray, A.K.: Color image segmentation: Rough-set theoretic approach. Pattern Recognition Letters 29, 483-493 (2008)

7. Panjwani, D.K., Healey, G.: Markov random field models for unsupervised segmentation of textured color images. IEEE Trans. Pattern Anal. Mach. Intell. 17(10), 939-954 (1995)

8. Pawlak, Z.: Rough sets. Int. J. of Inf. and Comp. Sci. 5, 341-356 (1982)

9. Pawlak, z.: Granularity of knowledge, indiscernibility and rough sets. In: Proceedings of IEEE International Conference on Fuzzy Systems, pp. 106-110 (1998)

10. Sen, D., Pal, S.K.: Generalized Rough Sets, Entropy, and Image Ambiguity Measures. IEEE Trans. Sys. Man and Cyb. 39(1), 117-128 (2009)

11. Petrosino, A., Ferone, A.: Feature Discovery through Hierarchies of Rough Fuzzy Sets. In: Chen, S.M., Pedrycz, W. (eds.) Granular Computing and Intelligent Systems: Design with Information Granules of Higher Order and Higher Type (to appear, 2011)

12. Shafarenko, L., Petrou, M., Kittler, J.V.: Histogram based segmentation in a perceptually uniform color space. IEEE Trans. Image Process. 7(9), 1354-1358 (1998)

13. Trémeau, A., Colantoni, P.: Regions adjacency graph applied to color image segmentation. IEEE Trans. Image Process. 9(4), 735-744 (2000)

14. Uchiyama, T., Arbib, M.A.: Color image segmentation using competitive learning. IEEE Trans. Pattern Anal. Mach. Intell. 16(12), 1197-1206 (1994)

15. Unnikrishnan, R., Pantofaru, C., Hebert, M.: A Measure for Objective Evaluation of Image Segmentation Algorithms. In: Proc. CVPR WEEMCV (2005)

16. Zadeh, L.A.: Fuzzy Sets. Information and Control 8, 338-353 (1965) 